\title{
Mitos del amor romántico y calidad de las relaciones sentimentales adolescentes
}
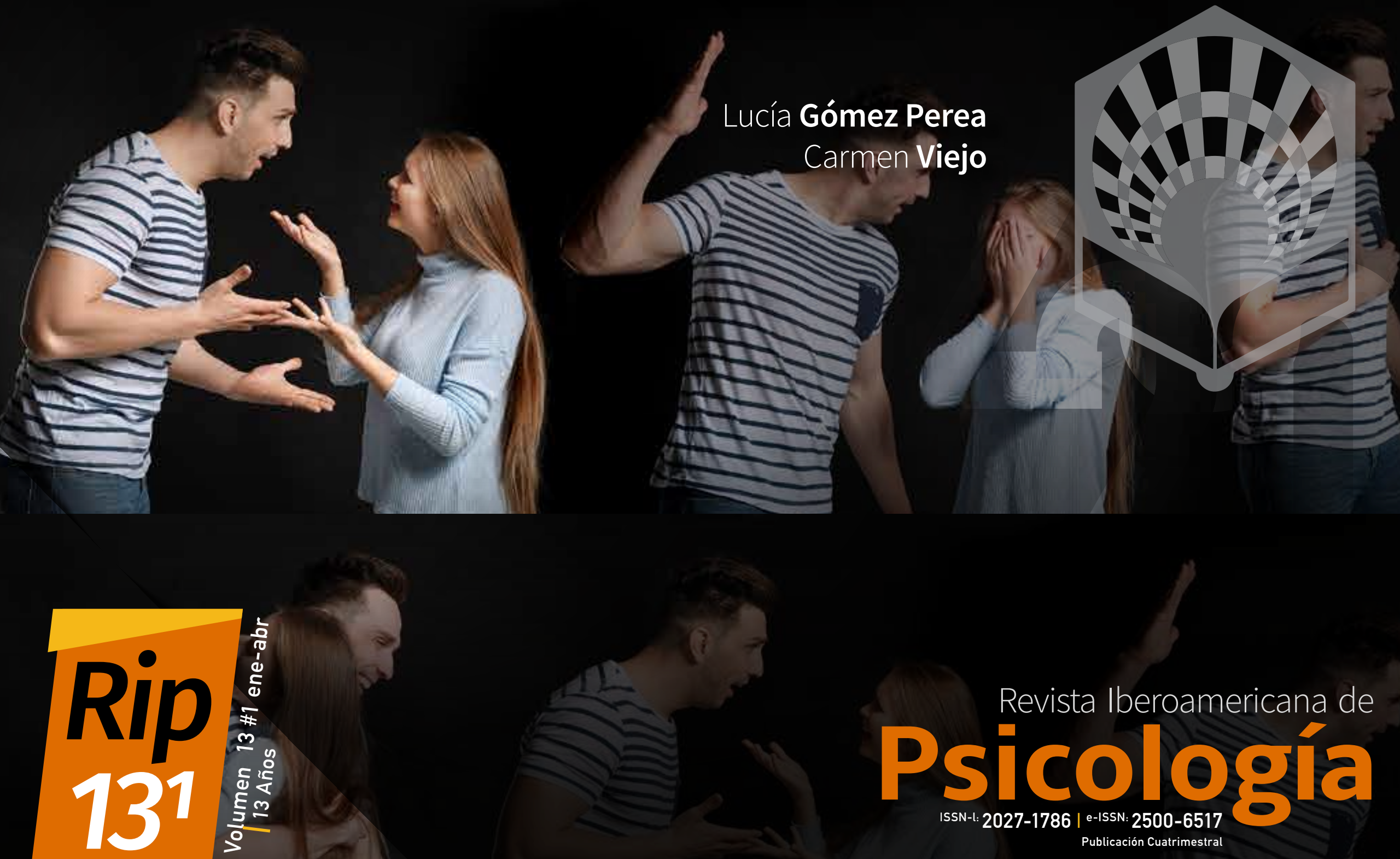
Revista Iberoamericana de

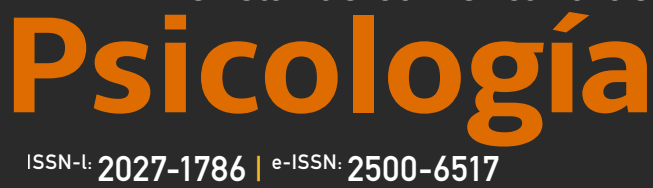

Publicación Cuatrimestral

ID:

10.33881/2027-1786.RIP.13114

Title: Myths of romantic love and quality of teen's relationships

Título: Mitos del amor romántico y calidad de las relaciones sentimentales adolescentes

Alt Title / Título alternativo:

[en]: Myths of romantic love and quality of teen's relationships

[es]: Mitos del amor romántico y calidad de las relaciones sentimentales adolescentes

Author (s) / Autor (es):

Gómez Perea \& Viejo

\section{Keywords / Palabras Clave:}

[en]: Romantic ideology; beliefs; romantic myths; dating; quality of couple; violence in couple; adolescents

[es]: Ideario romántico; creencias románticas; mitos; calidad de pareja; violencia en pareja; adolescentes.

Submited: 2019-06-14

Acepted: $\quad$ 2020-02-19

\section{Resumen}

La existencia de violencia en las relaciones de pareja adolescente es un fenómeno de creciente interés en el ámbito científico. El desarrollo de comportamientos agresivos en el interior de las primeras relaciones de intimidad dependerá en gran medida del ideario de amor que los y las adolescentes tengan y utilicen para modelar sus propias parejas; en este sentido, los mitos de amor romántico juegan un papel clave. Este estudio analiza el ideario romántico de los jóvenes, señalando las diferencias significativas en función al sexo y la edad; concretamente, así como la relación existente entre estos mitos y la calidad de las primeras parejas, identificando, a su vez, el grado de implicación en violencia que estos presentan. Participaron 583 estudiantes (49.6\% chicas), con edades comprendidas entre los 14 y los 18 años. Los resultados mostraron diferencias significativas en cuanto al ideario amoroso, en relación al sexo y a la edad de los participantes: las chicas se encontraban de acuerdo en mayor grado con los mitos referidos a la duración de la pasión de los primeros momentos y, al amor ciego; mientras que los chicos, con los mitos referidos a los celos como prueba de amor y la omnipotencia del amor verdadero; además, el grado de aceptación de los mitos aumentaba a medida que avanzaban en los años adolescentes. Fue posible identificar tres perfiles de ideario romántico, que se relacionaban con la implicación en violencia en la pareja: El amor justifica violencia, Baja idealización del amor e Ideal romántico no violento

\section{Abstract}

The existence of violence in adolescent relationships is a phenomenon of growing interest in the scientific field. The development

of aggressive behaviors within the first

relationships of intimacy to a large extent of the ideology of love that adolescents have and use to model their own partners; in this sense, the myths of romantic love play a key role. This study analyzes the romantic ideology of young people, pointing out the significant differences according to sex and age; specifically, as well as the relationship between these and the quality of the first couples, identifying, sometimes, the degree of involvement in the violence they present. 583 students participated $(49.6 \%$ girls), with ages between 14 and 18 years. The results, the differences, the differences in terms

of the love index, in the relationship with the sex and the age of the participants: the girls are in the most advanced degree with the myths referring to the duration of the passion of the first moments and, to the love of the blind; while the boys, with the myths referred to jealousy as proof of love and the omnipotence of love; In addition, the degree of acceptance of the myths increased as they progressed in the teenage years. It was possible to identify three profiles of romantic ideology, which is related to the involvement in violence in the couple: Love justifies violence, Low idealization of love and, The non-violent romantic ideal.

\section{Citar como:}

Gómez Perea, L. \& Viejo, C. (2020). Mitos del amor romántico y calidad de las relaciones sentimentales adolescentes. Revista Iberoamericana de Psicología, 13 (1) 151-161. Obtenido de: https:// reviberopsicologia.ibero.edu.co/article/view/1625

\section{Lucía Gómez Perea, MA Edu}

Source | Filiacion:

Universidad de Córdoba

BIO:

Graduada en Educación Infantil. Máster en Psicología Aplicada a la Educación y al Bienestar Social. Ha desarrollado investigaciones en torno a relaciones interpersonales, concretamente en el inicio del proceso de cortejo y las relaciones sentimentales adolescentes, así como los posibles riesgos asociados a estas.

City $\mid$ Ciudad:

Córdoba [es]

e-mail:

m22gopel@uco.es
Carmen Viejo

ORCID: $\quad$ https://orcid.org/0000-0003-4456-4767

Source | Filiacion:

Universidad de Córdoba

BIO:

Doctora, docente investigadora y miembro del Laboratorio de Estudios para la Convivencia y Prevención de la Violencia. Con intereses investigativos en relaciones interpersonales en la adolescencia, particularmente el inicio del proceso de cortejo y las relaciones sentimentales. Desde una mirada psicoevolutiva, ha estudiado los riesgos asociados a estas relaciones, tales como el dating violence, así como en sus beneficios y su relación con el bienestar psicológico y subjetivo durante el periodo adolescente.

City | Ciudad:

Córdoba [es]

e-mail:

cviejo@uco.es 


\section{Mitos del amor romántico y calidad de las relaciones sentimentales adolescentes}

Myths of romantic love and quality of teen's relationships

Lucía Gómez Perea

Carmen Viejo

\section{Introducción}

La adolescencia es un periodo de maduración y construcción social para los chicos y chicas en el que se suceden numerosos cambios físicos, psicológicos y sociales (Viejo \& Ortega Ruiz, 2015) algunos de los cambios más importantes son los relacionados con las nuevas demandas de rol de género y sexualidad que enfrentan en estas edades (Viejo, Romera, \& Vargas, 2020) y que impactan directamente en la evolución de la entidad del grupo de iguales y, particularmente, en la aparición de las primeras relaciones de intimidad o encuentros de interés erótico-sentimental. Conocer el ideario amoroso con el que los y las adolescentes enfrentan esta tarea evolutiva permitiría profundizar en el análisis de su impacto en la calidad de las relaciones sentimentales que se configuren, así como en su posible relación con determinados comportamientos violentos de baja intensidad que parecen estar muy presentes en estas primeras relaciones en cuanto más alto es el nivel de aferramiento a los denominados Mitos del Amor Romántico. 


\section{Marco teórico}

Las primeras relaciones sentimentales son, en la mayoría de los casos, el resultado obtenido de la evolución de una amistad entre iguales (Musitu \& Cava, 2003) y tendrán un papel esencial en el desarrollo adolescente, cumpliendo funciones afiliativas de apego, apoyo y cuidado, así como de satisfacción de necesidades e intereses sexuales (Viejo \& Ortega Ruiz, 2015). Sin embargo, no son relaciones exentas de cierto riesgo. Distintos autores han señalado que estos primeros acercamientos están caracterizados por un proceso de cortejo rudo y torpe, denominado dirty dating (Ortega \& Sánchez, 2011; Sánchez, Ortega Rivera, Ortega Ruiz, \& Viejo, 2008), que, sin embargo, suele ser bien considerado y aceptado entre los y las adolescentes que pueden interpretarlo como muestra de interés por parte de quien lo lleva a cabo (Viejo, 2012). Pueden distinguirse algunas diferencias entre las formas utilizadas por chicos y chicas, pues mientras que ellos prefieren las formas físicas más rudas, como empujones, agarrones, etc., ellas utilizan formas verbales como insultos o bromas irónicas (Viejo, Romera, \& Vargas, 2020). Pese a que esta pauta de comportamiento e interacción social podría formar parte de las dinámicas de acercamiento propias del cortejo en estas edades, también podría estar sentando las bases para que se consoliden estos comportamientos violentos en el interior de las primeras relaciones íntimas, llegando a formar parte de la dinámica de la relación. Ante este riesgo, los estudios han puesto de manifiesto que un alto porcentaje de chicos y chicas se ven envueltos en sus primeras parejas en dinámicas violentas que inciden negativamente en su bienestar y ajuste personal (Ortega \& Sánchez, 2011; Sánchez, Ortega Rivera, Ortega Ruiz, \& Viejo, 2008), en un patrón relacional agresivo denominado dating violence.

Kanin (1957) realizó un primer estudio en el que señaló que un $\mathbf{6 2} \%$ de las estudiantes universitarias afirmaban haber sido agredidas por sus parejas durante la etapa anterior a la universidad. Desde entonces, numerosos estudios se han venido desarrollando sobre este fenómeno reportando índices de prevalencia que han despertado las alarmas de la comunidad científica (Abilleira \& Rodicio-García, 2017; Jennings, y otros, 2017; Sánchez, Ortega Rivera, Ortega Ruiz, \& Viejo, 2008). El estudio de revisión sistemática de Jennings et al. (2017) sobre violencia de pareja entre chicos y chicas de 15 a 30 años, ha señalado que la prevalencia de esta violencia era menor $(<10 \%)$ entre los chicos y chicas más jóvenes que entre los más mayores ( 20-30\%), siendo ellas quienes informaban de una mayor prevalencia de victimización en comparación con ellos. Un estudio reciente realizado en España (Abilleira \& Rodicio-García, 2017), pone en evidencia que la violencia verbal es la más extendida entre los y las adolescentes, no obstante, existen ciertas diferencias entre chicas y chicos: ellas sufren más amenazas y violencia física, mientras que los chicos manifiestan sufrir más la violencia relacional.

Pese a estas tasas de violencia, distintos estudios han señalado que los chicos y chicas adolescentes perciben, de modo muy extendido, que sus relaciones son satisfactorias y tienen una buena calidad, entendiendo esta como el grado en que los miembros de la pareja muestran intimidad, afecto y apoyo mutuo (Barber, Crouter, \& Booth, 2006; Furman \& \& Shaffer, 2003). En general, las parejas que perciben una relación de pareja de alta calidad manifiestan mayores niveles de bienestar, satisfacción, compromiso y relaciones futuras más positivas (Collins, Welsh, \& Furman, 2009). No obstante, actualmente siguen sin estar delimitados los factores intrapersonales e interpersonales que establecen dicha calidad por completo (Martínez-Álvarez, FuertesMartín, Orgaz-Baz, Vicario-Molina, \& González-Ortega, 2014). Algunos estudios han apuntado que uno de los posibles factores que incidirían en esta interpretación sería la idea de pareja y relación sentimental que los chicos y chicas hayan construido (Martínez-Álvarez, Fuertes-Martín, Orgaz-Baz, Vicario-Molina, \& González-Ortega, 2014) señalando la idealización del amor y el amor romántico como elementos de riesgo para la formación de las relaciones de intimidad.

La sociedad, desde tiempos inmemorables, viene asimilado el concepto de amor y amor romántico como un sentimiento universal, histórico, inalterable y perpetuo, sin embargo, los estudios han puesto de manifiesto la incidencia que tiene el contexto sociocultural determinado en el que se consolide este sentimiento (Pascual, 2016) Concretamente, en la sociedad occidental, individualista y capitalista, se ha educado y socializado a la ciudadanía, en cuanto al amor se refiere, desde una mirada patriarcal, en la que la hombría se relaciona con la fuerza, la razón, la actividad, la independencia, el poder, la esfera pública, la autoridad y la libertad sexual, dando lugar a un hombre que vive para sí mismo y para que le sirvan. Por el contrario, la feminidad se encuentra relacionada con la fragilidad, la afectividad, la pasividad, el cuerpo y lo natural, dominio de los sentimientos, el ámbito privado y la capacidad de cuidar (Simón, 2009). De esta socialización, denominada socialización diferencial, se deriva el ideal de lo que se espera de chicos y chicas de forma diferenciada (Pascual, 2016), una idea de amor en la que las chicas asumen un papel de inferioridad respecto a los deseos del hombre y las necesidades del hogar, mientras que los chicos aprenden a ser el sustento económico familiar (Pascual, 2016). Para las chicas adolescentes, por tanto, el amor romántico se convierte en la búsqueda, la entrega, la fusión con la otra persona, la ansiedad y el compromiso. Este amor se convierte en una forma de organizar su futuro, elaborando su identidad personal (Leal, 2007). Para ellas, la atención y el cuidado hacia la pareja es uno de los aspectos que más favorece la afirmación del yo personal dentro de una relación, de forma que la atención a las necesidades del otro se convierte en la vía para sentirse valorada y querida (Leal, 2007). Por el contrario, para los chicos el amor involucra cierta ganancia sin comprometer aspectos del yo personal, lo que se vincula, además, con la seducción y el acceso al sexo femenino (Leal, 2007).

Este ideal de amor romántico da como resultado una serie de mitos, socialmente aceptados, que representan un "conjunto de creencias socialmente compartidas sobre la supuesta verdadera naturaleza del amor y, al igual que sucede en otros ámbitos, también suelen ser ficticios, absurdos, engañosos irracionales e imposibles de cumplir" (Yela, 2003). Entre los mitos identificados, la literatura científica ha subrayado el papel que juegan en estas primeras relaciones de intimidad el mito de la media naranja -la creencia de que existe una única persona en el mundo destinada a ser nuestra pareja ideal-; el mito del emparejamiento, entendido como la creencia de que acabar emparejado (pareja heterosexual) es algo natural y universal, y que la monogamia está presente en todos los tiempos y culturas; el mito de los celos que defiende que los celos son un buen indicador de que existe amor en una relación y que sirve como justificación de actitudes egoístas, injustas, represivas e incluso violentas; el mito de la omnipotencia, según el cual el amor verdadero lo puede todo y es más fuerte que cualquiera de los obstáculos que puedan surgir; o el mito de la pasión eterna o de la perdurabilidad, que defiende que el amor pasional de las primeras fases de la pareja debe perdurar para siempre con la misma intensidad (Yela, 2000; Yela, 2003). Que los chicos y las chicas asuman este ideario amoroso puede derivar en problemas relacionados con el ajuste personal o con la dinámica de la propia relación; dando lugar a relaciones demasiado exigentes que desemboquen en decepción, a la tolerancia o normalización de ciertos comportamientos poco ajustados, o a la incorporación de distintas manifestaciones de violencia como algo común dentro de la pareja (Ferrer, Bosch, \& Navarro, 2010). 
En nuestro país, diversos estudios han señalado la amplia aceptación que tienen estos mitos (Ferrer, Bosch, \& Navarro, 2010). El estudio de Ferrer et al. (2010), señaló que la mayoría de las personas en la etapa de juventud y adultez (edad media de $\mathbf{4 8 . 6 3}$ años) tienen una alta aceptación de mitos como la omnipotencia o la pasión eterna: entre el $\mathbf{7 0} \%$ y el $\mathbf{7 5 \%}$ de los participantes estaban de acuerdo con ideas como que el amor verdadero lo puede todo, o que la pasión intensa de los primeros tiempos de una relación debería durar siempre; y entre el $\mathbf{5 0 \%}$ y el $\mathbf{6 5 \%}$ afirmaban que el amor es ciego, o que en alguna parte hay alguien predestinado para cada persona (tu media naranja). No obstante, existían diferencias en relación al sexo de los participantes, siendo que ellas seguían creyendo que el amor lo puede todo en mayor proporción que ellos, mientras que los chicos empezaban a concederle mayor importancia a la pareja, y consideraban la ruptura como un fracaso en tasas más altas que las chicas (Ferrer, Bosch, \& Navarro, 2010). También la edad establecía algunas diferencias: las personas de 18 a $\mathbf{3 4}$ años mostraban significativamente más indiferencia hacia el mito del matrimonio y más aceptación del mito de la pasión eterna; las personas de $\mathbf{1 8}$ a $\mathbf{4 4}$ años mostraban significativamente más desacuerdo con el mito del emparejamiento; las personas de $\mathbf{3 5}$ a 44 años mostraban significativamente más indiferencia hacia la creencia de que el amor verdadero lo puede todo (mito de la omnipotencia); las personas de $\mathbf{4 5}$ a 64 años mostraban significativamente más desacuerdo con los mitos de la pasión eterna y la omnipotencia; y las personas de 65 o más años mostraban significativamente más indiferencia hacia el mito de la media naranja y más acuerdo con la creencia de que el amor verdadero lo puede todo y con el mito del emparejamiento.

Estos resultados ponen de manifiesto la importancia de seguir avanzando con estudios que analicen, particularmente en las edades adolescentes y juveniles, el ideario amoroso de los chicos y chicas en relación a sus primeras relaciones íntimas, así como la incidencia que este pueda tener en la percepción de la calidad de sus parejas e incluso en la implicación en ciertos comportamientos violentos como parte de la dinámica de la relación. En este sentido, el presente trabajo pretende contribuir a este cuerpo de literatura, dando respuesta a los siguientes objetivos: 1) analizar cuál es la idea de amor actual entre los y las jóvenes en relación a los mitos del amor romántico; 2) analizar las diferencias que el sexo y edad de los participantes pudiera establecer en el concepto de amor que tengan; y 3) examinar la relación y posible incidencia que la aceptación de los mitos del amor romántico puede tener en la calidad de las parejas de los y las adolescentes y su implicación en comportamientos violentos.

\section{Método}

\section{Población y muestra}

Se llevó a cabo un muestreo incidental por accesibilidad que dio como resultado una muestra inicial de $\mathbf{6 1 1}$ participantes, estudiantes de Educación Secundaria de $\mathbf{3}$ centros de la ciudad de Córdoba (2 de titularidad pública y 1 concertada). Dado que el rango que edad ascendía en algunos a periodos evolutivos fuera del objeto de estudio, se llevó a cabo una selección de aquellos chicos y chicas con edades comprendidas entre los $\mathbf{1 4}$ y $\mathbf{1 8}$ años (edad media $\mathbf{1 6 . 2 3}$ años; D.T.= $\mathbf{1 . 0 0}$ ), siendo eliminados el $\mathbf{4 . 8 \%}$ de los y las participantes.

La muestra final quedó definida por $\mathbf{5 8 3}$ estudiantes (49.6\% chicas y $\mathbf{5 0 . 4 \%}$ chicos), escolarizados en Educación Secundaria Obligato- ria (3o ESO: $n=54,9.5 \%$; 4을 ESO: $n=155,27.3 \%$ ), Bachillerato (10 curso: $n=159,28.0 \%$; 20 curso: $n=131,23.1 \%)$; estudios de Grado Medio ( $n=53 ; 9.3 \%)$ y Formación Básica $(n=16 ; 2.7 \%)$.

\section{Diseño implementado}

Este trabajo es un estudio de tipo descriptivo mediante encuestas; con un diseño transversal, pues las variables han sido analizadas en un momento dado (Montero \& León, 2002).

\section{Procedimiento}

Se pasaron las encuestas al alumnado durante el último trimestre de 2017 (noviembre- diciembre). Se invertía una media de 30-45 minutos en la cumplimentación del cuestionario.

\section{Técnicas e Instrumentos de recolección de la información}

La batería de medida utilizada para este estudio estuvo compuesta por las siguientes escalas:

Dating Questionnaire (Connolly, Craig, Goldberg, \& Pepler, 1999; Connolly, Furman, \& Konarski, 2000). Este cuestionario recoge información sobre las actividades que los jóvenes realizan en su tiempo libre, el tiempo que le dedican a su pareja, el tipo de relación que mantienen en la actualidad y su duración. Se mide a través de preguntas abiertas, de tipo verdadero y falso y, de respuesta múltiple. Está compuesto de 9 ítems.

Adaptación de la Escala sobre Mitos del Amor Romántico de Bosch (Rodríguez-Castro, Lameiras, Carrera, \& Vallejo-Medina, 2013). El objetivo de este cuestionario es conocer la idea que los chicos y chicas pueden tener en relación al amor y las relaciones de pareja, Este instrumento se puntúa mediante una escala Likert, siendo 1 totalmente en desacuerdo y 5 totalmente de acuerdo a través de los 7 ítems que lo componen. Estos mitos van referidos a la existencia de "una media naranja”, la pasión que no tiene fin, la ceguedad del amor, celos como sinónimo de amor, relación entre amory maltrato, y amor todopoderoso. Siguiendo a Ferrer et al. (2010), se trabaja con cada uno de los ítems, sin constituir una escala propiamente.

Network Relationship Inventory -NRI-. (Furman \& Buhrmester, 1992), cuyo objetivo es conocer la relación actual que los adolescentes mantengan o hayan mantenido recientemente con su pareja. Hace referencia a comportamientos de carácter transgresivo para el mantenimiento de la pareja. Se puntúa mediante una escala Likert del 1 al 5. Siendo 1 "nunca" y 5 "siempre", a través de un total de 15 ítems. Este instrumento recoge 4 factores: Compañía $(\alpha=.76)$; Expectativa de futuro $(\alpha=.90)$; Comunicación $(\alpha=.82)$ y Conflicto $(\alpha=.87)$.

Adaptación del CADRI (Wolfe, y otros, 2001), fue elaborado específicamente para detectar la existencia de actos violentos en las relaciones de pareja adolescentes. Se mide a través una escala Likert, cuyas cinco opciones van desde "nunca" (esto no ha pasado en nuestra relación), hasta "siempre”. La adaptación utilizada (Viejo \& Ortega Ruiz, 2015), cuenta con un total de 18 ítems que reportan índices de agresión 
y victimización a través de 6 factores: violencia psicológica cometida ( $\alpha$ = .83); violencia psicológica sufrida ( $\alpha=$.87); agresión física moderada $(\alpha=$.78); agresión física severa $(\alpha=$.93); victimización física moderada $(\alpha=.81)$ y victimización física severa $(\alpha=.81)$.

\section{Análisis de la información}

Los datos fueron codificados e incorporados a una base de datos para su procesamiento con el programa de software estadístico SPSS 22. En primer lugar, se realizaron análisis descriptivos sobre la experiencia sentimental de los participantes, así como para la aceptación que podían tener de los mitos del amor romántico trabajados en este estudio en función al sexo y la edad de los mismos. Esto último se realizó a través de pruebas de contraste de proporciones ANOVA y pruebas T-Student, calculando el tamaño de efecto con la prueba Eta cuadrado y la d de Cohen respectivamente (Norman, 2010). En segundo lugar, se llevó a cabo un análisis de clúster o conglomerados para conocer los perfiles existentes en cuanto a los distintos tipos de creencias o mitos que podían tener los adolescentes. Se utilizó para ello un análisis de conglomerado bietápico, considerando los 7 ítems del instrumento de Rodríguez-Castro et al. (2013), como variables continuas, de tipo aglomerativo. Se llevó a cabo un primer análisis sin determinar el número de agrupaciones; tras estudiar los resultados, se llevó a cabo un segundo análisis delimitando a tres el número de grupos.

Tabla 1. Experiencia sentimental en función del sexo

\section{Consideraciones éticas asociadas al desarrollo del estudio}

Se solicitó a los centros educativos su participación en este estudio explicándoles los objetivos de la investigación y facilitándoles una copia del cuestionario que se usaría. Tras obtener los permisos y consentimientos necesarios tanto por parte de los padres y propios adolescentes como de los respectivos centros, y bajo los criterios éticos de confidencialidad y anonimato, cumpliendo con los requerimientos de la Declaración de Helsinki.

\section{Resultados}

En primer lugar, se describió la experiencia sentimental de los chicos y chicas participantes. Los resultados señalaron que solo un $\mathbf{2 2 . 3 \%}$ de los y las adolescentes no habían tenido ningún tipo de experiencia sentimental. El $\mathbf{3 6 . 8 \%}$ tenía algún tipo de relación en el momento de la encuesta y el $\mathbf{4 0 . 9 \%}$ la había tenido en el pasado, aunque ahora no la tuviera. En relación a la comparación por sexos, el estadístico Chicuadrado $\left(X^{2}(4, N=557)=21.94, p=.000 ; V=.02 ; \gamma=.098\right)$ señaló que existían diferencias significativas entre los chicos y las chicas a pesar de que el tamaño del efecto era bajo, siendo que ellas tenían más experiencia sentimental que ellos (Tabla 1).

\begin{tabular}{|c|c|c|c|}
\hline & Chicos & Chicas & Total \\
\hline En este momento salgo con alguien & $13.6 \%$ & $22.6 \%$ & $36.3 \%$ \\
\hline En este momento salgo con más de uno/a & $0.5 \%$ & $0.0 \%$ & $0.5 \%$ \\
\hline $\begin{array}{l}\text { En este momento no salgo con nadie, pero he } \\
\text { estado con alguien en los últimos dos meses }\end{array}$ & $7.0 \%$ & $6.3 \%$ & $13.3 \%$ \\
\hline $\begin{array}{l}\text { En este momento no salgo con nadie, pero he } \\
\text { estado con alguien hace más de dos meses }\end{array}$ & $16.0 \%$ & $11.7 \%$ & $27.6 \%$ \\
\hline Nunca he salido con nadie & $12.7 \%$ & $9.5 \%$ & $22.3 \%$ \\
\hline \multicolumn{4}{|c|}{$N=557$ Missing $=4.5 \%$} \\
\hline
\end{tabular}

\section{Idea de amor romántico: diferencias por sexo}

Con el objetivo de avanzar en la descripción del imaginario de pareja que tienen los chicos y chicas, se llevó a cabo un análisis descriptivo de las puntuaciones medias alcanzadas respecto a las posibles creencias que los y las adolescentes pudieran tener sobre el amor romántico, considerando además las posibles diferencias que establecieran el sexo y la edad de los participantes.

Los datos señalaron puntuaciones medias de aceptación de los mitos del amor que oscilaban entre 1.12 y 4.15. Aquellos mitos que ob- tenían mayores puntuaciones fueron: La pasión intensa de los primeros tiempos debería durar siempre, El amor verdadero lo puede todo, El amor es ciego y, En alguna parte hay alguien predestinado para cada persona ("tu media naranja"); mientras que los referidos a Los celos son una prueba de amor, Se puede amar a alguien a quien se maltrata y, Se puede maltratar a alguien a quien se ama obtuvieron las puntuaciones más bajas (ver Figura 1).

Respecto a la incidencia del sexo en estos resultados, el estadístico T-Student señaló diferencias significativas entre los chicos y las chicas en los ítems que se refieren a la duración de la pasión de los primeros momentos [t $(562)=-3.46 ; p=.00 ; r=-0.14]$ y al amor ciego [t $(562)=-2.21 ; p=.03 ; r=-0.09]$ siendo las chicas quienes puntúan más alto (4.15 vs.3.83; 3.53 vs. 3.28, respectivamente). Por otro lado, en 
los ítems que refieren los celos como prueba de amor [t (561) = 3.42; $p=.00 ; r=0.14]$ y la omnipotencia del amor verdadero $[t(566)=2.92$, $p=.00 ; r=0.10]$ son ellos los que obtienen unas puntuaciones significativamente más altas (2.13vs.1.80; 3.55 vs.3.23, respectivamente). Teniendo todas ellas un pequeño tamaño de efecto, pues su D de Cohen se encuentra por debajo de 0.20 .

Respecto a las diferencias establecidas en función de la edad, el estadístico de análisis de proporciones ANOVA y la prueba post-hoc Figura 1. Puntuaciones medias basadas en creencias sobre el amor romántico en función de la edad

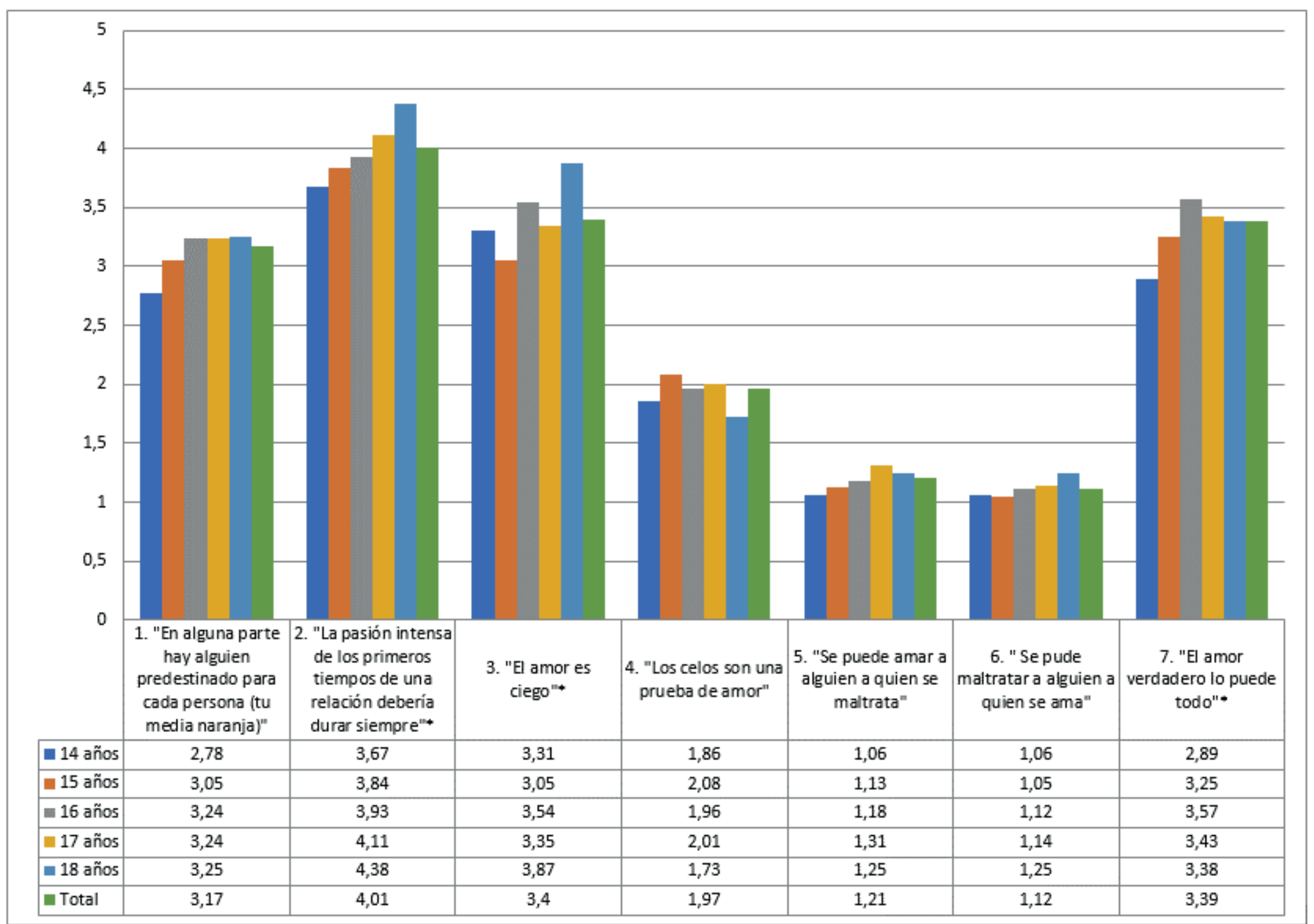

*. p. $\leq 05$

Fuente: Elaboración propia

\section{Perfiles de ideario romántico}

\section{y su relación con la calidad}

\section{de la pareja e implicación en violencia}

En segundo lugar, para identificar los posibles perfiles en función a las creencias que los chicos y chicas pueden tener respecto a los mitos del amor romántico, se llevó a cabo un análisis de conglomerados en dos fases o bietápico. Se establecieron como variables continuas los siete ítems de la adaptación del instrumento de Lameiras (2013). Como resultado se obtuvieron tres agrupaciones con una suficiente calidad establecida por una silueta de cohesión media de 0.3, donde el orden de los predictores fue: Se puede amar a alguien a quien se maltrata (1.00), Se puede maltratar a alguien a quien se ama (0.48), El amor es de Tukey señalaron diferencias estadísticamente significativas en varios de los ítems. Concretamente, en La pasión intensa de los primeros tiempos de una relación debería durar siempre $[F(4,572)=4.14 p=.00$, $\eta 2=0.03]$, (14, 15 y 16 años vs. 18 años); El amor es ciego [F(4, 572)= $4.93 p=.00, \eta 2=0.03$ ] (15 vs.16 años, 15 vs. 18 y, 17 vs. 18); $y, E l$ amor verdadero lo puede todo $[F(4,576)=2.44 p=.05, \eta 2=0.02],(14$ vs.16 años), siendo todas ellas débiles puesto que su Eta cuadrado se encuentra por debajo de 0.04 (ver Figura 1).
El primer grupo, el cual se denominó como El amor justifica violencia, fue el menos numeroso, compuesto por un $\mathbf{9 . 6 \%}$ de la muestra. Un $\mathbf{1 0 . 4 \%}$ eran chicos y un $\mathbf{8 . 5 \%}$ chicas, y tenían una edad media de $\mathbf{1 6 . 5 8}$ años. El segundo grupo estaba formado por un $\mathbf{4 0 . 4 \%}$ de la muestra y se denominó como Baja idealización del amor. Un $\mathbf{4 0 . 7 \%}$ eran chicos y un $\mathbf{4 1 . 0 \%}$ eran chicas, con una media de edad de $\mathbf{1 5 . 9 6 .}$ El tercer grupo, fue denominado como Ideal romántico no violento, y se encontraba compuesto por un $\mathbf{5 0 . 0 \%}$ de la muestra. Un $\mathbf{4 8 . 9 \%}$ eran chicos y un $\mathbf{5 0 . 6 \%}$ chicas, con una media de edad de $\mathbf{1 6 . 3 7}$ años.

Tras realizar pruebas de comparación de medias, se encontraron diferencias estadísticamente significativas en todos los mitos entre, al menos, dos de los grupos establecidos (Tabla 2). El grupo 1 se caracte- 


\section{Mitos del amor romántico y calidad de las relaciones sentimentales adolescentes}

rizó por tener puntuaciones significativamente más altas que los otros dos grupos en los ítems referidos a la aceptación de la violencia: Se puede amar a alguien a quien se maltrata, y Se puede maltratar a alguien a quien se ama. El grupo 2 se definió con los valores más bajos, de manera significativa, en todos los mitos respecto a los demás gru- pos, excepto en los de violencia que presentaba valores homólogos a los del grupo 3. Este último grupo se caracterizó por tener las puntuaciones significativamente más altas en todos los mitos, excepto en los de violencia, que compartía bajas puntuaciones con el grupo 2.

Tabla 2. Perfiles de ideal romántico

\begin{tabular}{|c|c|c|c|c|c|c|c|}
\hline & & $\begin{array}{l}\text { Clúster } 1 \text { Elamor } \\
\text { justifica violencia }\end{array}$ & $\begin{array}{c}\text { Clúster } 2 \text { Baja } \\
\text { idealización del } \\
\text { amor }\end{array}$ & $\begin{array}{c}\text { Clúster } 3 \text { Ideal } \\
\text { romántico no } \\
\text { violento }\end{array}$ & Total & Estadístico ANOVA & $\begin{array}{c}\text { Diferencias } \\
\text { entre grupos } \\
\quad 2 \mathrm{a} 2\end{array}$ \\
\hline \multirow{2}{*}{\multicolumn{2}{|c|}{ Tamaño }} & $9.6 \%$ & $40.4 \%$ & $50.0 \%$ & & & \\
\hline & & \multicolumn{4}{|c|}{-277} & & \\
\hline \multirow{2}{*}{ Sexo } & Chico & $10.4 \%$ & $40.7 \%$ & $48.9 \%$ & & & \\
\hline & Chica & $8.5 \%$ & $41.0 \%$ & \multicolumn{2}{|l|}{$50.6 \%$} & & \\
\hline Edad m & & 16.58 & 15.96 & 16.37 & & & \\
\hline \multirow{2}{*}{\multicolumn{2}{|c|}{ Item 11}} & \multirow{2}{*}{3.36} & \multirow{2}{*}{2.43} & \multirow{2}{*}{3.69} & \multirow{2}{*}{3.68} & \multirow{2}{*}{$\begin{array}{c}{[F(2,551)=75.08} \\
p=.00 ; \eta 2=.21]\end{array}$} & $1-2$ \\
\hline & & & & & & & $2-3$ \\
\hline \multirow{3}{*}{\multicolumn{2}{|c|}{ Item 22}} & \multirow{3}{*}{3.87} & \multirow{3}{*}{3.33} & \multirow{3}{*}{4.57} & \multirow{3}{*}{4.17} & \multirow{3}{*}{$\begin{array}{c}{[\mathrm{F}(2,551)=107.54} \\
\mathrm{p}=.00 ; \eta 2=.28]\end{array}$} & $1-2$ \\
\hline & & & & & & & $1-3$ \\
\hline & & & & & & & $2-3$ \\
\hline \multirow{3}{*}{\multicolumn{2}{|c|}{ Item 33}} & \multirow{3}{*}{3.40} & \multirow{3}{*}{2.51} & \multirow{3}{*}{4.09} & \multirow{3}{*}{3.70} & \multirow{3}{*}{$\begin{array}{c}{[F(2,551)=125.62 ;} \\
p=.00 ; \eta 2=.31]\end{array}$} & $1-2$ \\
\hline & & & & & & & $1-3$ \\
\hline & & & & & & & $2-3$ \\
\hline \multirow{2}{*}{\multicolumn{2}{|c|}{ Item 44}} & \multirow{2}{*}{2.45} & \multirow{2}{*}{1.46} & \multirow{2}{*}{2.27} & \multirow{2}{*}{2.09} & \multirow{2}{*}{$\begin{array}{c}{[\mathrm{F}(2,551)=41.54 ;} \\
\mathrm{p}=.00 ; \eta 2=.13]\end{array}$} & $1-2$ \\
\hline & & & & & & & $2-3$ \\
\hline \multirow{2}{*}{\multicolumn{2}{|c|}{ Item 55}} & \multirow{2}{*}{2.83} & \multirow{2}{*}{1.02} & ? & 140 & {$[F(2,551)=517.90$} & $1-2$ \\
\hline & & & & 1.03 & 1.48 & $p=.00 ; \eta 2=.65]$ & $1-3$ \\
\hline Itomer & & (2) & 107 & 100 & 1 ก & {$[F(2,551)=182.08$} & $1-2$ \\
\hline Item 66 & & 2.21 & 1.03 & 1.00 & 1.04 & $p=.00 ; \eta 2=.40]$ & $1-3$ \\
\hline $1+0 m 7$ & & 261 & 256 & 100 & S & {$[F(2,551)=99.23$} & $1-2$ \\
\hline rem ry & & 3.64 & 2.56 & 4.00 & 3.59 & $p=.00 ; \eta 2=.27]$ & $2-3$ \\
\hline 1 "En al & una part & ay alguien predestin & do para cada perso & ("tu media naranj & & & \\
\hline 2"La pa & ión inte & de los primeros tien & os de una relación & ebería durar siemp & & & \\
\hline 3 "El am & rescieg & & & & & & \\
\hline 4 "Los C & losson $\mathrm{c}$ & prueba de amor" & & & & & \\
\hline 5 "Se pu & de ama & guien a quien se $m$ & trata" & & & & \\
\hline 6 "Se pu & de malt & r a alguien a quien s & ama" & & & & \\
\hline 7 "El am & erdac & o puede todo" & & & & & \\
\hline
\end{tabular}

Finalmente, se estableció la relación entre estos perfiles y las variables calidad de pareja e implicación en violencia mediante un análisis de comparación de medias. Se encontraron diferencias estadísticamente significativas en tres de las escalas referentes a la calidad de pareja: Expectativa de futuro, Comunicación y, Conflicto, y, en cuatro de las referidas a implicación en violencia: Violencia psicológica cometida, Violencia psicológica sufrida, Agresión física moderada y, Victimización física severa, entre, al menos, dos de los grupos establecidos (Tabla 3). El grupo 1 se caracterizó por tener puntuaciones significativamente más altas que los otros dos grupos en las escalas referidas a la aceptación de la violencia: Conflicto, Violencia psicológica cometida, Violencia psicológica sufrida, Agresión física moderada y, Victimización física severa. El grupo 2 se definió con los valores más bajos en cuanto a su Expectativa de futuro y, Comunicación de manera significativa, respecto al grupo 3. Sin embargo, en cuanto a su implicación en violencia presentaba valores homólogos a este, excepto en la escala referida a Violencia psicológica cometida, donde el tercer grupo se veía mayormente implicado. Este último grupo se caracterizó por tener las puntuaciones significativamente más altas en todas las escalas referidas a una calidad de pareja positiva. 
Tabla 3. Calidad e implicación en violencia de los perfiles

\begin{tabular}{|c|c|c|c|c|c|c|c|}
\hline & & $\begin{array}{c}\text { Clúster } 1 \\
\text { El amor justifica } \\
\text { violencia }\end{array}$ & $\begin{array}{c}\text { Clúster } 2 \\
\text { Baja idealización } \\
\text { del amor }\end{array}$ & $\begin{array}{c}\text { Clúster } 3 \\
\text { Ideal romántico } \\
\text { no violento }\end{array}$ & Total & $\begin{array}{l}\text { Estadístico } \\
\text { ANOVA }\end{array}$ & $\begin{array}{c}\text { Diferencias } \\
\text { entre grupos } \\
\quad 2 \text { a } 2\end{array}$ \\
\hline \multirow{4}{*}{ 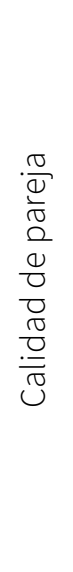 } & Compañía & 3.80 & 3.87 & 4.05 & 3.79 & $\begin{array}{l}{[F(2,389)=2.61 ;} \\
p=.07 ; \eta 2=.01]\end{array}$ & \\
\hline & Expectativa de futuro & 3.39 & 3.08 & 3.58 & 3.32 & $\begin{array}{c}{[F(2,378)=7.05} \\
p=.01 ; \eta 2=.04]\end{array}$ & $2-3$ \\
\hline & Comunicación & 3.72 & 3.50 & 3.99 & 3.60 & $\begin{array}{l}{[F(2,385)=9.84} \\
p=.00 ; \eta 2=.05]\end{array}$ & $2-3$ \\
\hline & Conflicto & 2.66 & 2.14 & 2.29 & 2.78 & $\begin{array}{l}{[F(2,377)=5.24} \\
p=.01 ; \eta 2=.03]\end{array}$ & $\begin{array}{l}1-2 \\
2-3\end{array}$ \\
\hline \multirow{6}{*}{ 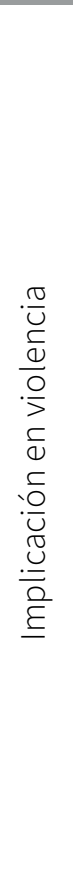 } & $\begin{array}{c}\text { Violencia psicológica } \\
\text { cometida }\end{array}$ & 2.00 & 1.57 & 1.74 & 1.88 & $\begin{array}{l}{[\mathrm{F}(2,381)=8.14} \\
\mathrm{p}=.00 ; \eta 2=.04]\end{array}$ & $\begin{array}{l}1-2 \\
1-3 \\
2-3 \\
\end{array}$ \\
\hline & $\begin{array}{c}\text { Violencia psicológica } \\
\text { Sufrida }\end{array}$ & 2.23 & 1.69 & 1.84 & 2.01 & $\begin{array}{l}{[F(2,386)=8.48} \\
p=.00 ; \eta 2=.04]\end{array}$ & $\begin{array}{l}1-2 \\
1-3\end{array}$ \\
\hline & $\begin{array}{c}\text { Agresión física } \\
\text { Moderada }\end{array}$ & 1.18 & 1.13 & 1.11 & 1.25 & $\begin{array}{l}{[F(2,393)=3.42 ;} \\
p=.03 ; \eta 2=.02]\end{array}$ & $1-3$ \\
\hline & Agresión física Severa & 1.22 & 1.07 & 1.07 & 1.10 & $\begin{array}{c}{[F(2,393)=2.33} \\
p=.09 ; \eta 2=.01]\end{array}$ & \\
\hline & $\begin{array}{l}\text { Victimización física } \\
\text { Moderada }\end{array}$ & 1.34 & 1.20 & 1.18 & 1.27 & $\begin{array}{l}{[F(2,393)=1.61} \\
p=.20 ; \eta 2=.01]\end{array}$ & \\
\hline & $\begin{array}{c}\text { Victimización física } \\
\text { severa }\end{array}$ & 1.34 & 1.06 & 1.10 & 1.13 & $\begin{array}{l}{[F(2,393)=6.78 ;} \\
p=0.01 ; \eta 2=.03]\end{array}$ & $\begin{array}{l}1-2 \\
1-3\end{array}$ \\
\hline
\end{tabular}

Fuente: Elaboración propia

\section{Discusión y conclusiones}

Las primeras relaciones de pareja adolescente conforman una de las estructuras sociales más importantes de este periodo de la vida, pues son clave dentro de la compleja vida social de chicos y chicas, afectando al plano de su identidad e intimidad, satisfaciendo necesidades de afecto, y desarrollando su autoconcepto. Los resultados de este estudio señalaron que alrededor de un $\mathbf{7 7 . 7 \%}$ de los participantes tenían en la actualidad o habían tenido pareja en alguna ocasión anterior, lo que evidencia que la pareja adolescente es ya un contexto relacional significativo en la mayoría de los adolescentes (Sánchez, Ortega Rivera, Ortega Ruiz, \& Viejo, 2008; Van de Bongardt, Yu, Deković, \& Meeus, 2015). Estas primeras relaciones, que forman parte del desarrollo normativo, marcan ciertas pautas que acompañarán a los y las adolescentes en sus futuras relaciones a lo largo de la vida. Pese al alto porcentaje de adolescentes que tienen experiencia sentimental, el carácter incipiente de estas relaciones, unido a la falta de experiencia sentimental previa por parte de sus protagonistas pueden dar lugar a relaciones ineficaces, plagadas de malas estrategias de pareja y, en los casos más graves, con comportamientos violentos integrados como parte de la dinámica de relación. En muchas ocasiones son las altas tasas de conflictos existente que la literatura científica ha señalado a lo largo de los últimos años (Abilleira \& Rodicio-García, 2017; Jennings, y otros, 2017; Sánchez, Ortega Rivera, Ortega Ruiz, \& Viejo, 2008) los desencadenantes de estas situaciones. Si bien, actualmente existe un amplio acuerdo en identificar esta violencia como un fenómeno multicausal y multifactorial, para cuya intervención es preciso abordar diferentes elementos.

En este sentido, y pese a no estar totalmente definidos los factores intrapersonales e interpersonales que determinan la calidad de estas parejas (Martínez-Álvarez, Fuertes-Martín, Orgaz-Baz, VicarioMolina, \& González-Ortega, 2014) algunos estudios señalan a la idealización del amor y el amor romántico como uno de los factores que incidirían en la interpretación del ideal de pareja y de la relación sentimental (Martínez-Álvarez, Fuertes-Martín, Orgaz-Baz, Vicario-Molina, \& González-Ortega, 2014) serían, por tanto, elementos de riesgo en la formación de estas relaciones.

Los dos primeros objetivos de este estudio trataban de analizar cuál es la idea de amor actual entre los y las jóvenes en relación a los mitos del amor romántico, teniendo en cuenta el sexo y la edad de los participantes. Al respecto, los resultados revelaron que los mitos más aceptados por ambos sexos en general fueron los referidos a la duración incondicional del amor, por encima del tiempo y las dificultades, pese a existir ligeras diferencias entre chicos y chicas: ellas se encontraban de acuerdo en mayor grado con los mitos referidos a la duración de la pasión de los primeros momentos y, al amor ciego. Mientras que ellos, obtuvieron puntuaciones más altas de aceptación de los mitos referidos a los celos como prueba de amor y la omnipotencia del amor verdadero. Estos resultados contrastan con los referidos por el estudio de Ferrer et al. (2010), que señalaba que eran las chicas las más estereo- 


\section{Mitos del amor romántico y calidad de las relaciones sentimentales adolescentes}

tipadas en cuanto al ideario de amor, y quienes más creían que el amor lo puede todo. En cuanto a las diferencias significativas halladas en función de la edad de los participantes, se encontró con que los mitos de La pasión intensa de los primeros tiempos de una relación deberían durar siempre, El amor es ciego y, El amor verdadero lo puede todo, ganaban aceptación conforme los chicos y chicas iban alcanzando edades más avanzadas en los tres casos. Algunos de estos resultados contrastan con los referidos por el estudio de Ferrer et al. (2010), pues la creencia en la pasión eterna disminuye en los participantes de mayor edad con respecto a los menores. Sin embargo, en el caso de la omnipotencia del amor, sí que coinciden los resultados ya que en dicho estudio fueron los participantes más longevos quienes se mostraron mayormente de acuerdo con este.

El tercero de los objetivos trataba de identificar los posibles perfiles en función a la aceptación de los mitos del amor romántico y la relación y posible incidencia que puede tener en la calidad de las parejas de los y las adolescentes, así como en su implicación en comportamientos violentos. Al respecto, se identificaron tres perfiles que se denominaron: el amor justifica violencia, baja idealización del amor e, ideal romántico no violento, en función de sus características. A raíz del establecimiento de estos tres grupos, los resultados mostraron que, un alto nivel de aceptación de los mitos del amor romántico aumentaba el grado de implicación en violencia y a su vez disminuía la calidad de la pareja levemente. Mientras que, por el contrario, un bajo ideal romántico, disminuía el grado de implicación en violencia, aumentando la calidad de la pareja con respecto al primer grupo. Sin embargo, esta calidad no sufría grandes cambios respecto de un grupo a otro. Los resultados coincidieron con los obtenidos anteriormente en el estudio llevado a cabo por Jennings et al. (2017) en cuanto a la edad, pues el grupo mayormente relacionado con la violencia era el que tenía una mayor media de edad. Por otro lado, se puede observar como la violencia psicológica es la que predomina en los tres grupos, resultado que coincide con el recogido en el estudio de Abilleira y Rodicio- García (2017) el cual constataba que la violencia verbal era la más extendida entre su muestra de adolescentes.

Este estudio avanza en la línea de investigación sobre violencia dentro de las relaciones de pareja adolescente y contribuye aportando resultados significativos respecto a la relación existente entre posibles creencias sobre el amor que estos tienen interiorizadas y la calidad e incluso implicación en violencia a la que pueden dar lugar. Sin embargo, es preciso tener a considerar algunas limitaciones como la imposibilidad de establecer relaciones causales que profundicen en la explicación del fenómeno y permitan establecer modelos explicativos, debido a que se ha tratado de un estudio de carácter transversal o, el tamaño reducido de la muestra y su carácter incidental; haciendo imposible que esta sea representativa y, por tanto, los resultados no puedan generalizarse a otras poblaciones. Por lo que sería interesante ahondar en esta línea con estudios longitudinales que permitan tener varias medidas temporales del fenómeno y conocer su evolución; además se podría llevar a cabo con una muestra que sí sea representativa en cuanto a edad, sexo o cualquier otra variable que se considere de interés.

\section{Referencias}

Abilleira, M. P., \& Rodicio-García, M. L. (2017). Análisis del autoconcepto en las víctimas de violencia de género entre adolescentes. Suma psicológica, 24(2), 107-114. doi:10.1016/j.sumpsi.2017.08.001

Barber, B. L., Crouter, A. C., \& Booth, A. (2006). To have loved and lost: Adolescent romantic relationships and rejection. Romance and sex in adolescence and emerging adulthood: Risks and opportunities, 29-40. doi:10.4324/9781410617361
Collins, W. A., Welsh, D. P., \& Furman, W. (2009). Adolescent romantic relationship. Annual review of psychology,, 60, 631-652. doi:10.1146/ annurev.psych.60.110707.163459

Connolly, J., Craig, W., Goldberg, A., \& Pepler, D. (1999). Conceptions of cross-sex friendships and romantic relationships in early adolescence. Journal of Youth and Adolescence, 28(4), 481-494. doi:https://doi.org/10.1023/A:1021669024820

Connolly, J., Furman, W., \& Konarski, R. (2000). The role of peers in the emergence of heterosexual romantic relationships in adolescence. Child development, 71(5), 1395-1408. doi:https://doi.org/10.1111/14678624.00235

Ferrer, V. A., Bosch, E., \& Navarro, C. (2010). Los mitos románticos en España. Boletín de psicología(99), 7-31. Obtenido de https://www. researchgate.net/profile/Victoria Ferrer-Perez/publication/46311908 Los mitos romanticos en Espana/links/Ofcfd50c3334483eb5000000/ Los-mitos-romanticos-en-Espana.pdf

Furman, W., \& \& Shaffer, L. (2003). The Role of Romantic Relationships in Adolescent Development. En P. Florsheim, \& P. Florsheim (Ed.), Adolescent romantic relations and sexual behavior (Primera ed., págs. 17-36). Nueva York: Psychology Press. doi:10.4324/9781410607782

Furman, W., \& Buhrmester, D. (1992). Age and sex differences in perceptions of networks of personal relationships. Child development, 63(1), 103115. doi:https://doi.org/10.1111/j.1467-8624.1992.tb03599.x

Jennings, W. G., Okeem, C., Piquero, A. R., Sellers, C. S., Theobald, D., \& Farrington, D. P. (2017). Dating and intimate partner violence among young persons ages 15-30: Evidence from a systematic review. Aggression and violent behavior, 33, 107-125. doi:10.1016/j. avb.2017.01.007

Kanin, E. (1957). Male Aggresion in Dating-Courtship Relations. American Journal of Sociology, 63(2), 197-204. doi:10.1086/222177

Leal, A. (2007). Nuevos tiempos, viejas preguntas sobre el amor. Un estudio con adolescentes. Posgrado y Sociedad, 7(2), 50-70. Obtenido de https://dialnet.unirioja.es/servlet/articulo?codigo $=3662521$

Martínez-Álvarez, J. L., Fuertes-Martín, A., Orgaz-Baz, B., Vicario-Molina, I., \& González-Ortega, E. (2014). Vínculos afectivos en la infancia y calidad en las relaciones de pareja de jóvenes adultos: el efecto mediador del apego actual. Anales de psicología, 30(1), 211-220. doi:10.6018/ analesps.30.1.135051

Montero, I., \& León, O. G. (2002). Clasificación y descripción de las metodologías de investigación en Psicología. International journal of clinical and health psychology, 2(3), 503-508. Obtenido de https:// wWW.redalyc.org/pdf/337/33720308.pdf

Musitu, G., \& Cava, M. J. (2003). El rol del apoyo social en el ajuste de los adolescentes. Intervención psicosocial, 12 (2), 179-192. Obtenido de https://wWw.redalyc.org/pdf/1798/179818034005.pdf

Norman, G. (2010). Likert scales, levels of measurement and the "laws" of statistics. Advances in health sciences education, 15(5), 625-632. doi:https://doi.org/10.1007/s10459-010-9222-y

Ortega, R., \& Sánchez, V. (2011). Juvenile dating and violence. En C. Monks, \& L. Coyne, Bullying in Different Contexts (págs. 113-136). New York: Cambridge University Press.

Pascual, A. (2016). Sobre el mito del amor romántico. Amores cinematográficos y educación. Dedica. Revista de educação e humanidades, 10, 63-78. Obtenido de https://digibug.ugr.es/ handle/10481/41940

Rodríguez-Castro, Y., Lameiras, M., Carrera, M. V., \& Vallejo-Medina, P. (2013) Validación de la Escala de Actitudes hacia el Amor en una muestra de adolescentes. Estudios de Psicología, 34(2), 209-219. doi:https://doi. org/10.1174/021093913806751429

Sánchez, V., Ortega Rivera, F., Ortega Ruiz, R., \& Viejo, C. (2008). Las relaciones sentimentales en la adolescencia: satisfacción, conflictos y violencia. Escritos de Psicología (Internet), 2(1), 97-109. Obtenido de http://scielo.isciii.es/scielo.php?script=sci arttext\&pid $=$ S1989-38092008000300011 
Simón, N. (2009). Del mito del amor romántico a la construcción de relaciones amorosas entre iguales: una mirada feminista. En A. Gil, G. Escrig, \& A. Forcada, V Actas Congreso. Poder, poderes y empoderamiento... ¿Y el amor? ¡Ah, el amor! (págs. 51-62). Castellón: Publicacions de la Universitat Jaume I. Servei de Comunicació i Publicacions. Obtenido de http://repositori.uji.es/xmlui/. handle/10234/84709

Van de Bongardt, D., Yu, R., Deković, M., \& Meeus, W. (2015). Romantic relationships and sexuality in adolescence and young adulthood: The role of parents, peers, and partners. European Journal of Developmental Psychology, 15(2), 497-515. doi:https://doi.org/10.1080/ 17405629.2015.1068689

Viejo, C. (2012). Dating violence y cortejo adolescente. Un estudio sobre la violencia en las parejas sentimentales de los jóvenes andaluces. Tesis doctoral, Universidad de Córdoba, España. Obtenido de https:// helvia.uco.es/handle/10396/7642

Viejo, C., \& Ortega Ruiz, R. (2015). Cambios y riesgos asociados a la adolescencia. Psychology, Society, \& Education, 7(2), 109-118. doi:10.25115/psye.v7i2.527
Viejo, C., Romera, E., \& Vargas, S. (2020). Mejora de la calidad de las relaciones sentimentales adolescentes a través del desarrollo de la competencia social: el programa parejas cosim como propuesta de intervención. Paideia, revista de educación(57), 11-41. Obtenido de http://revistasacademicas.udec.cl/index.php/paideia/article/view/1494

Wolfe, D. A., Scott, K., Reitzel-Jaffe, D., Wekerle, C., Grasley, C., \& Pittman, A L. (2001). Development and validation of the conflict in adolescent dating relationships inventory. Psychological Assessment, 13(2), 277293. doi:https://doi.org/10.1037/1040-3590.13.2.277

Yela, C. (2000). El amor desde la perspectiva de la Psicología Social. Madrid: Pirámide.

Yela, C. (2003). La otra cara del amor: mitos, paradojas y problemas. Encuentros en Psicología Social, 1(2), 263-267. doi:10.13140/ RG.2.1.4142.7289 